\title{
Toxicity of Chimeric Antigen Receptor T Cells and its Management
}

\author{
Kitsada Wudhikarn', Shui Yen Soh², He Huang ${ }^{3}$, Miguel-Angel Perales $^{4,5}$
}

'Division of Hematology and Research Unit in Translational Hematology, Faculty of Medicine, Chulalongkorn University, Bangkok, Thailand, 'Paediatric Haematology/Oncology Service, KK Women's and Children's Hospital, Singapore, ${ }^{3}$ The First Affiliated Hospital, School of Medicine, Zhejiang University, Hangzhou, China, ${ }^{4}$ Adult Bone Marrow Transplant Service, Department of Medicine, Memorial Sloan Kettering Cancer Center, New York, New York, USA, ${ }^{5}$ Department of Medicine, Weill Cornell Medical College, New York, NY, USA

\begin{abstract}
Recently, chimeric antigen receptor (CAR) T cell therapy has transformed the treatment armamentarium of relapsed/refractory B lymphoid malignancies. CAR T cells provide an excellent response rate and potential cure for these patients. However, CAR T cells also possess unique and potentially life-threatening immune-mediated side effects. Among these, cytokine release syndrome (CRS) and immune effector cell-associated neurotoxicity syndrome (ICANS) are the two most common complications associated with CAR T cell therapy. While the pathogenesis of CRS involves the activation of complex immune axes, including both cellular networks and inflammatory cytokine milieu, the mechanism of ICANS has not been fully elucidated. Other notable toxicities of CAR T cells include macrophage activation syndrome, cytopenia, and potential organ toxicities. Treatments for these complications typically encompass close observation, multidisciplinary supportive measures, and cytokinemodifying agents such as anti-interleukin- 6 antibody and systemic corticosteroids. CAR T therapies can cause immunologic adverse events and management of these toxicities could also instigate a profound immune suppression state that predisposes patients to a variety of infectious complications. Prompt diagnosis and proper management of these complications are crucial to minimize CAR T cell-associated complications and to maximize the outcome of CAR T cell therapy.
\end{abstract}

Key words chimeric antigen receptor T cell, CAR T cell, cytokine release syndrome, CRS, immune effector cell associated neurotoxicity syndrome, ICANS, infection

Submitted August 7, 2021; Accepted August 15, 2021; Published online October 14, 2021; Issued online October 14, 2021

Correspondence Author: Kitsada Wudhikarn, Division of Hematology, Faculty of Medicine, Chulalongkorn University, Bangkok, Thailand, E-mail: Kitsada.w@chula.ac.th

This article was created from selected presentations at the 26th Annual Congress of APBMT and was handled by Guest Editor Shinichiro Okamoto before submission.

\section{Introduction}

Chimeric antigen receptor (CAR) $\mathrm{T}$ cells are engineered $\mathrm{T}$ cells that are transduced to express an artificially modified receptor that directly binds to target antigens on tumor cells. The engagement between CAR T cells and tumor cells results in a supraphysiologic immune reaction, which eventually results in tumor cell eradication. However, this overwhelming immunological derangement can also lead to other on-target, off-tumor immune-mediated complications. Cytokine release syndrome (CRS) and immune effector cell-associated neurotoxicity syndrome (ICANS) are the two most prominent adverse events of CAR T cell therapy. Other than ICANS and CRS, prolonged cytopenia, B-cell/plasma cell aplasia, and hypogammaglobulinemia are wellestablished complications. These adverse effects can result in a profound immunocompromised state that predisposes patients to various infectious complications. Unlike hematopoietic stem cell transplantation, little is 
known about the dynamics of immune reconstitution after CAR T cell therapy, including appropriate antimicrobial utilization and immunizations for these patients. This review will focus on the biology and proper treatments of these common complications, which will help to improve outcomes for patients who undergo CAR T cell therapy.

\section{Biology of CAR T Cell Toxicities}

CAR $\mathrm{T}$ cell therapy is a form of engineered adoptive cellular therapy that has shown remarkable efficacy in the treatment of cancer, especially hematologic malignancies. The efficacy of this therapy depends on the CAR T cells' recognition of the target tumor-associated antigen, followed by CAR $\mathrm{T}$ cell activation, expansion, and immune-killing of the cancer cells, as well as sufficient persistence to provide immune surveillance for tumor control. This $\mathrm{T}$ cell immune response in turn sets the stage for the toxicities that frequently accompany CAR T cell therapies, namely CRS and ICANS ${ }^{1}$.

\section{Biology of CRS}

CRS is a systemic hyperinflammatory response syndrome characterized by fever with or without hypotension, hypoxia, or other organ dysfunction ${ }^{1}$. Factors predictive of CRS severity include high disease burden, use of a fludarabine/cyclophosphamide ("FluCy") lymphodepleting (LD) chemotherapy regimen, high CAR-T cell dose, and rapid/robust CAR-T cell expansion in vivo $^{2}$. C-reactive protein (CRP) and serum ferritin are common laboratory markers that reflect systemic inflammation and are elevated during $\mathrm{CRS}^{3}$. Preclinical and clinical studies have shown elevated levels of various cytokines in $\mathrm{CRS}^{3-6}$. Activated CAR T cells produce interferon (IFN)-gamma, tumor necrosis factor (TNF) alpha, and granulocyte-macrophage colony-stimulating factor (GM-CSF). These cytokines activate and stimulate myeloid cells (monocytes and macrophages) to produce interleukin-6 (IL-6), IL-1, and IL-10, which are the key mediators (especially IL-6) that drive the manifestations of CRS. Correspondingly, treatment of CRS using the IL-6 receptor antagonist tocilizumab has been shown to produce rapid and efficacious responses ${ }^{7}$.

Secondary hemophagocytic lymphohistiocytosis (sHLH) or macrophage activation syndrome (MAS) is a potentially life-threatening complication that can occur following CAR T cell therapy. The clinical and laboratory features of CAR-T-associated sHLH/MAS overlap substantially with CRS, reflecting the activation of the reticuloendothelial system initiated by $\mathrm{T}$ cell-mediated inflammation. Therefore, sHLH/MAS likely represents the severe fulminant end of the spectrum of systemic hyperinflammation rather than a distinct entity ${ }^{1}$.

\section{Biology of ICANS}

Compared to CRS, the biology of ICANS is less clearly understood. ICANS commonly occurs after CRS, and the onset and severity of ICANS are correlated with those of CRS. Manifestations of ICANS include encephalopathy, decrease in the level of consciousness, seizures, motor weakness, and cerebral edema with raised intracranial pressure ${ }^{1}$. Factors predictive of severe ICANS include high tumor burden, high CAR-T cell dose, higher peak CAR-T cell expansion in vivo, severe and earlier-onset CRS, and pre-existing neurologic comorbidities ${ }^{8,9}$. There is an overlap in the clinical and laboratory inflammatory profiles of ICANS and CRS. Elevated levels of cytokines (IL-1, IL-6, IL-2, GM-CSF) and inflammatory cells in the systemic circulation and cerebrospinal fluid (CSF) have been correlated with the development of ICANS in animal models and clinical studies, ${ }^{4,10}$. However, ICANS does not respond well to tocilizumab treatment, suggesting that IL6 is not a dominant player in the pathophysiology of ICANS $^{4}$. Clinical evidence of endothelial dysfunction (such as capillary leak syndrome, thrombocytopenia, and disseminated intravascular coagulation [DIC]) and elevated serum levels of biomarkers of endothelial activation (angiopoietin-2; ANG2 and von Willebrand Factor; VWF) have been observed in patients with severe ICANS $^{8}$. Endothelial dysfunction/activation and blood brain barrier (BBB) disruption, possibly mediated by systemic elevation in inflammatory cytokines (IL-1, IL6 , TNF- $\alpha$ ), with infiltration of both CAR and non-CAR $\mathrm{T}$ cells into the central nervous system (CNS), have therefore been identified as potential mechanisms contributing to ICANS $^{8-10}$. Elevated levels of inflammatory cytokines in the CSF are also correlated with increased concentrations of endogenous excitatory neurotransmitters, such as glutamate (Glut) and quinolinic acid (QA) in ICANS $^{9}$.

\section{Biology of other CAR-T cell toxicities}

Other CAR-T cell toxicities include on-target offtumor effects and prolonged cytopenia. While persistence of adequate CAR T cells is desired for better tumor control, this would also result in persistent ontarget off-tumor effects. B cell aplasia and hypogammaglobulinemia are therefore the anticipated on-target off-tumor effects of B-cell-directed CAR T cell therapies. The biology of prolonged cytopenia after CAR T cell therapy is less well understood but may be caused by an LD preparatory regimen or extensive prior cytotoxic therapies. A review of post-CAR-T hematopoietic recovery in a large series of patients showed that delayed recovery of blood counts is correlated with higher-grade CRS or ICANS, as well as with higher levels of vascular endothelial growth factor and 
macrophage-derived chemokines ${ }^{11}$.

\section{Immune-mediated Toxicities of CAR T Cell Ther- apy: Manifestations, Assessments, and Manage- ment}

CAR T cell therapy is considered an advanced and effective immunotherapy, especially for hematological malignancies, and at the time of writing this manuscript, five CAR $\mathrm{T}$ cell products (axicabtagene ciloleucel $^{12}$, tisagenlecleucel ${ }^{13}$, brexucabtagene autoleucel $^{14}$, lisocabtagene maraleucel ${ }^{15}$ and idecabtagene vicleucel $^{16}$ ) have already been approved by the FDA. However, these CAR-T cell products possess a series of toxicities, which accompany their powerful anti-tumor activities. With the increasing number of clinical trials focused on CAR T cell therapy, an increasing amount of knowledge has been gained regarding the management of the associated toxicities. Meanwhile, accumulating evidence for the management of toxicities after CAR $\mathrm{T}$ cell therapy greatly facilitates the practice of CAR T cell therapy in itself.

\section{Presentations of CRS}

The main manifestations of CRS include fever, hypotension, hypoxemia, tachycardia, multi-organ dysfunction, and coagulation disorders. Several grading systems for CAR-T cell toxicities have been published by different groups. These include the National Cancer Institute (Common Terminology Criteria for Adverse Events, CTCAE), Lee et al. ${ }^{17}$, University of Pennsylvania $^{18}$, Memorial Sloan Kettering Cancer Center ${ }^{19}$, and the MD Anderson Cancer Center (CAR T cell-therapyassociated TOXicity, CARTOX group ${ }^{20}$. In addition, the consensus of the American Society for Transplantation and Cellular Therapy (ASTCT) has divided the grade of CRS into four levels according to the severity of fever, hypotension, and hypoxia ${ }^{1}$.

The grade of CRS should be considered for the management and treatment of the toxicity. The administration of non-steroidal anti-inflammatory drugs and the evaluation of infectious pathogens are fundamental therapeutic strategies. Tocilizumab should be administered for persistent high fever. Furthermore, fluid replacement is necessary when hypotension occurs, and the use of vasoactive drugs should also be considered depending on the severity of hypotension. Glucocorticoids must be used in severe CRS (grade 3-4), and patients with grade 4 CRS should be transferred to the intensive care unit for close monitoring.

\section{Presentations of ICANS}

Neurotoxicity caused by CAR $\mathrm{T}$ cell therapy is probably caused either by hyperproduction of cytokines that diffuse into the brain or by CAR $T$ cells that pass through the blood-brain barrier $(\mathrm{BBB})^{20}$. Published studies have shown that the symptoms of ICANS include headache, encephalopathy, delirium, anxiety, tremor, dizziness, aphasia, ataxia, seizure, dyscalculia, and myoclonus. To assess ICANS severity, ASTCT has created a new grading system of ICANS that combines the immune effector cell-associated encephalopathy (ICE) score (modified version of CARTOX-1 ${ }^{20}$ ) with clinical manifestations, findings of electroencephalography, and neuroimaging ${ }^{1}$. Notably, the classification of ICANS for children $<12$ years is different, replacing ICE with the Cornell Assessment for Pediatric Delirium instead ${ }^{21}$.

The treatment of ICANS should be based on symptoms, supportive therapies including low doses of lorazepam or haloperidol should be administered for anxiety, and corticosteroids and mannitol should also be administered for brain edema. Moreover, monitoring using neuroimaging and diagnostic lumbar puncture is important. Seizures should be controlled by an anticonvulsant under the guidance of neurologists.

\section{Hematologic toxicities}

Cytopenia occurred in all blood cell lineages and was observed in approximately $32 \%$ of the patients (lasting more than 28 days) after CAR T cell therapy ${ }^{22}$. The underlying mechanisms of cytopenia are not fully understood, but it is plausible that cytokines may hamper the differentiation of hematopoietic stem cells. It is worth noting that persistent neutropenia would lead to severe infections, which should be emphasized. Prophylaxis of infection plays a fundamental role, while blood transfusions and the use of granulocyte-colony stimulating factor (G-CSF) are also critical.

HLH/MAS is characterized by a hyperactivation of macrophages and lymphocytes and an abnormal elevation of various cytokines, often seen during CRS or the recovery period of CRS, resulting in multi-organ failure. The differential diagnosis of HLH from CRS is sometimes difficult because of the overlapping clinical manifestations and laboratory findings ${ }^{23}$. Current treatments in clinical practice include anti-IL-6 therapy and corticosteroids $^{24}$, and systemic therapy according to the HLH-2004 guidelines could be considered when no clinical and laboratory improvement is observed $\mathrm{d}^{24,25}$.

\section{Infectious Complications, Immune Reconstitution, and Prophylactic Strategies after CAR T Cell Therapy}

As stated in the previous section, CAR T cells can potentially cause several adverse effects. Among these potential complications, serious infections due to hypogammaglobulinemia, B-cell aplasia, and prolonged 


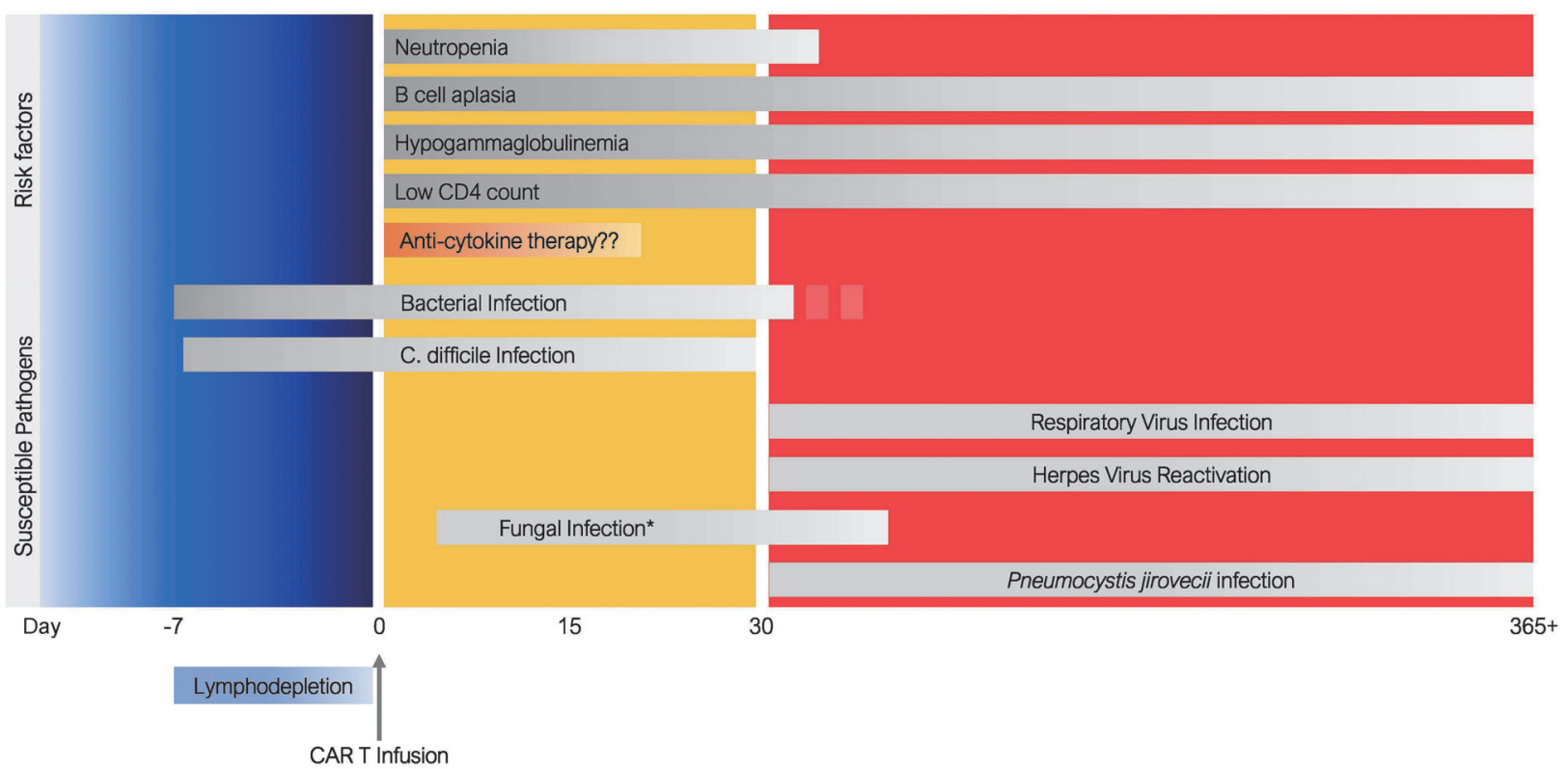

Figure 1. Predisposing factors and susceptible pathogens for infectious complication at different stages of CD19 chimeric antigen receptor $\mathrm{T}$ cell therapy

cytopenia may impede the success of CAR T cell therapy $^{26}$. Understanding the characteristics and risk factors of infection, including immune kinetics associated with CAR T cell therapy, will result in improved patient outcomes.

\section{Incidence and characteristics of infection after CAR T cell therapy}

Most data on infection following CAR T cell therapy have been derived from single-center retrospective studies in patients treated with CD19 CAR T cells, in addition to some data from prospective pivotal clinical trials. Currently, information about infection after BCMAtargeted CAR T cell therapy remains limited.

Patients with hematologic malignancy who undergo CD19 CAR T cell therapy can develop infection at several time points after treatment. Infection associated with CAR $\mathrm{T}$ cell therapy may be divided into three phases: the initiation of LD chemotherapy, early postCAR T cell therapy (day 0 to +30 ), and late phase after CAR $T$ cell therapy (beyond day +30 to +365 days or beyond) (Figure 1) ${ }^{27}$. The pattern of infection and dominant causative pathogens during each period is different and is attributed to the primary component of immune suppression at specific time points. Most studies showed a similar incidence and pattern of infection after CAR T cell therapy. The incidence of infection, especially severe infections and bacterial infections, is more common during the first 30 days. After the early phase, most infections are mild to moderate in severity, and the virus becomes more problematic compared to bacterial infection. Fungal infection is relatively uncom- mon, but can be observed in patients with severe and prolonged neutropenia.

\section{Risk factors of infection in CAR T cell therapy}

Infections in patients treated with CAR $\mathrm{T}$ cells result from factors including underlying host immunity, primary hematological diagnosis, and CAR-T cell specific effects, as well as the interactions among these factors. We can simply divide the underlying predisposing factors for infection in these patients into host-related and CAR-T cell-related factors. Underlying host immuneassociated factors may include prior treatments, primary hematologic malignancy, and underlying medical conditions. CAR T-associated factors that were reported to be associated with infection in this patient population include, but are not limited to, CAR-T cell dose, target of CAR-T cell product, LD chemotherapy-associated cytopenia, severe CRS/ICANS, and cytokine-directed therapy for CRS/ICANS,

\section{Antimicrobial prophylaxis and immunoglobulin re- placement in CAR T cell therapy}

There are several professional organizations and expert opinion statements that provide recommendations on prophylactic and management strategies for infection after CAR $\mathrm{T}$ cell therapy ${ }^{27-29}$. Here, we highlight prophylactic antimicrobial approaches for patients who undergo CD19-targeted CAR T cell therapy.

Most guidelines recommend initiating antibacterial prophylaxis during the severe neutropenia period with absolute neutrophil counts lower than $0.5 \times 10^{9} / \mathrm{L}$ and to continue it until absolute neutrophil count stays sus- 
tained above this level ${ }^{29}$. The most common antibiotic option for antibacterial prophylaxis is fluoroquinolone (such as levofloxacin). In addition to antibiotic prophylaxis, most experts recommend considering G-CSF in patients with prolonged neutropenia as clinically indicated $^{30,31}$.

Prophylactic acyclovir is recommended from the initiation of LD chemotherapy for herpes viral prophylaxis. Most guidelines recommend maintaining acyclovir prophylaxis for an extended period of time or adopting a CD4-guided approach to continue anti-viral prophylaxis until CD4 lymphocyte counts are higher than 200/ $\mu$ L. Patients who are hepatitis B carriers (HBs Ag-positive) or have a previous history of hepatitis B infection (HBsAg negative, anti-HBc Ab IgG positive), should receive entecavir for at least 6 months along with surveillance by liver function test or HBV DNA.

Although fungal infection is uncommon in patients undergoing CD19 CAR T cell therapy, anti-fungal prophylaxis should be considered in some patients with prolonged cytopenia or those requiring prolonged systemic corticosteroids for immune-mediated adverse events. In patients who are not at a high risk of fungal infection and do not have a previous history of active fungal infection, fluconazole may suffice and should be continued until the resolution of neutropenia. However, azoles with a broader anti-fungal spectrum may be necessary in patients with prolonged neutropenia or prior mold infection. For pneumocystis infection prophylaxis, most guidelines recommend trimethoprim/sulfamethoxazole to be initiated at around 1 month post-CAR-T infusion if blood count recovery allows. Otherwise, other alternatives with no potential effect on cytopenia, such as atovaquone or inhaled pentamidine should be considered.

As CD19, CD22, and other antigens targeted by CAR $\mathrm{T}$ cells are widely expressed by B cells, B cell aplasia is an unavoidable off-target toxicity (or depletion of plasma cells in patients receiving BCMA CAR $\mathrm{T}$ cell therapy), which results in hypogammaglobulinemia. Routine examination of immunoglobulin levels after CAR T cell therapy should not be neglected. Currently, data on intravenous immunoglobulin (IVIG) replacement in CAR T cell therapy are extrapolated from patients with hematologic malignancy who received anti-CD20 monoclonal antibodies, however, it is unclear whether IVIG replacement alters the overall post-CAR $\mathrm{T}$ cell IgG level or improves survival outcomes. Practice patterns on IVIG replacement can vary widely among practitioners and institutions. Currently, most guidelines recommend IVIG replacement for patients with IgG levels below $400 \mathrm{mg} / \mathrm{dL}$ or between 400 and $600 \mathrm{mg} / \mathrm{dL}$ for those who have recurrent infections ${ }^{27,32}$.

\section{Immunization in patients after CAR T cell therapy}

Most guidelines recommend starting immunization with inactivated/killed pathogen vaccines after 3-6 months and consider administering a live attenuated virus vaccine at least 12 months after CAR-T cell (or until CD4 lymphocyte count is $\geq 200 / \mu \mathrm{L}$ ). Physicians may incorporate pathogen-specific IgG levels and postimmunization immune responses to guide vaccination decisions in these patients. Table 1 summarizes the recommendations for vaccination of patients treated with CAR T cells.

Infectious complications are common in patients undergoing CAR T cell therapy. As CAR T cell therapy will become a critical treatment component of hematologic malignancy, a better understanding of the natural history of infection and the kinetics of immune reconstitution in these patients will provide physicians with more insight to provide proper management for patients.

\section{Conclusion}

CAR $\mathrm{T}$ cell therapy is a powerful and promising form of cancer immunotherapy, but it can be associated with significant toxicities, namely CRS and ICANS, and delayed hematologic recovery. Understanding biology means understanding the immunology of immune effector cell therapy and the complex interplay of therapy factors (e.g., CAR T cells, lymphodepletion), disease factors (e.g., diagnosis, tumor burden), and patient factors. Better strategies are needed to improve treatment efficacy and to prevent, monitor, and treat various CAR T-associated toxicities.

As CAR T cell therapy becomes the standard of care treatment option for patients with hematologic malignancies and has the potential to move forward to the earlier treatment setting, we will increasingly encounter both acute and late-onset CAR T-associated complications. Several research studies investigating the underlying mechanism of CAR T-related toxicities, predictive biomarkers, and novel interventions for these complications are actively ongoing and will hopefully lead to an individualized approach, better overall treatment experience, and outcomes in our patients.

\section{Author Contributions}

K.W., S.Y.S., H.H., and MAP concepted and wrote the article.

\section{Conflicts of Interest}

K.W., S.Y.S. and H.H. declare no relevant conflict of interest. M.A.P. reports honoraria from Abbvie, Astellas, 
Table 1. Vaccination for adult patients after CD19 chimeric antigen receptor T cell therapy (modified from Hill and Seo. Blood. 2020 Aug 20; 136 (8): 925-935)

\begin{tabular}{|c|c|c|c|c|c|c|c|c|c|c|c|c|c|c|}
\hline $\begin{array}{l}\text { Killed/inactivated } \\
\text { vaccines } \#\end{array}$ & $\begin{array}{l}\text { Pre- } \\
\text { CAR }\end{array}$ & $\geq 6 \mathrm{~m}$ & $\geq 6 \mathrm{~m}$ & $\geq 7 \mathrm{~m}$ & $\geq 8 \mathrm{~m}$ & $\geq 10 \mathrm{~m}$ & $\geq 12 \mathrm{~m}$ & $\geq 18 \mathrm{~m}$ & $\geq 20 \mathrm{~m}$ & $\geq 22 \mathrm{~m}$ & $\geq 24 \mathrm{~m}$ & $\geq 26 \mathrm{~m}$ & $\geq 27 \mathrm{~m}$ & $\begin{array}{c}\text { Minimal time } \\
\text { interval } \\
\text { between doses }\end{array}$ \\
\hline Influenza (inactivated) & $x$ & & $x$ & & & & & & & & & & & \\
\hline Pneumococcal conjugate & & Titer & $x$ & Titer $^{*}$ & $x$ & $x$ & & & & & & & & $1-2 m$ \\
\hline $\begin{array}{l}\text { Pneumococcal } \\
\text { polysaccharide }\end{array}$ & & Titer & & Titer $^{*}$ & & & & $x$ & Titer* & & & & & \\
\hline $\begin{array}{l}\text { Diphtheria/Tetanus/ } \\
\text { acellular Pertussis }\end{array}$ & & Titer & $x$ & Titer $^{*}$ & $x$ & $x$ & Titer* & & & & & & & $1-2 m$ \\
\hline $\begin{array}{l}\text { Hemophilus influenza } \\
\text { type B }\end{array}$ & & Titer & $x$ & Titer $^{*}$ & $x$ & $x$ & & Titer ${ }^{*}$ & & & & & & $1-2 m$ \\
\hline Hepatitis A & & Titer & $x$ & Titer $^{*}$ & & & $x$ & Titer* & & & & & & $6 \mathrm{~m}$ \\
\hline Hepatitis B & & Titer & $x$ & Titer $^{*}$ & $x$ & & $x$ & Titer* & & & & & & $2 \mathrm{~m}$ \\
\hline $\begin{array}{l}\text { Live and non-live } \\
\text { adjuvant Vaccines } \$\end{array}$ & $\begin{array}{l}\text { Pre- } \\
\text { CAR } \\
\end{array}$ & $6 \mathrm{~m}$ & $6+m$ & $7+m$ & $8+m$ & $10+m$ & $12+m$ & $18+m$ & $20+m$ & $22+m$ & $24+m$ & $26+m$ & $27+m$ & $\begin{array}{c}\text { Time between } \\
\text { doses }\end{array}$ \\
\hline $\begin{array}{l}\text { Mump-Measle-Rubella } \\
\text { (MMR) }\end{array}$ & & & & & & & & $x$ & & $x$ & & & Titer $^{*}$ & \\
\hline $\begin{array}{l}\text { Varicellar-Zoster (live); } \\
\text { Seronegative }\end{array}$ & & & & & & & & $\mathrm{x}$ & & $\mathrm{x}$ & & & & $1 \mathrm{~m}$ \\
\hline $\begin{array}{l}\text { Varicellar-Zoster (non-live } \\
\text { adjuvant) in VZV seroposi- } \\
\text { tive patients, }>50 \text { years }\end{array}$ & & & & & & & & $x$ & & $x$ & & & & $1-2 m$ \\
\hline
\end{tabular}

Bristol-Myers Squibb, Celgene, Equilium, Incyte, Karyopharm, Kite/Gilead, Merck, Miltenyi Biotec, MorphoSys, Novartis, Nektar Therapeutics, Omeros, Takeda, and VectivBio AG, Vor Biopharma. He serves on DSMBs for Cidara Therapeutics, Medigene, Sellas Life Sciences, and Servier, and the scientific advisory board of NexImmune. He has ownership interests in NexImmune and Omeros. He has received research support for clinical trials from Incyte, Kite/Gilead, Miltenyi Biotec, and Novartis. He serves in a volunteer capacity as a member of the Board of Directors of the American Society for Transplantation and Cellular Therapy (ASTCT) and Be The Match (National Marrow Donor Program, NMDP), as well as on the CIBMTR Cellular Immunotherapy Data Resource (CIDR) Executive Committee. Disclosure forms provided by the authors are available on the website.

\section{References}

1. Lee DW, Santomasso BD, Locke FL, Ghobadi A, Turtle CJ, Brudno JN, et al. ASTCT Consensus Grading for Cytokine Release Syndrome and Neurologic Toxicity Associated with Immune Effector Cells. Biol Blood Marrow Transplant. 2019; 25: $625-38$

2. Hay KA, Hanafi LA, Li D, Gust J, Liles WC, Wurfel MM, et al. Kinetics and biomarkers of severe cytokine release syn- drome after CD19 chimeric antigen receptor-modified T-cell therapy. Blood. 2017; 130: 2295-306.

3. Teachey DT, Lacey SF, Shaw PA, Melenhorst JJ, Maude SL, Frey N, et al. Identification of Predictive Biomarkers for Cytokine Release Syndrome after Chimeric Antigen Receptor Tcell Therapy for Acute Lymphoblastic Leukemia. Cancer Discov. 2016; 6: 664-79.

4. Norelli M, Camisa B, Barbiera G, Falcone L, Purevdorj A, Genua M, et al. Monocyte-derived IL-1 and IL-6 are differentially required for cytokine-release syndrome and neurotoxicity due to CAR T cells. Nat Med. 2018; 24: 739-48.

5. Giavridis T, van der Stegen SJC, Eyquem J, Hamieh M, Piersigilli A, Sadelain M. CAR T cell-induced cytokine release syndrome is mediated by macrophages and abated by IL-1 blockade. Nat Med. 2018; 24: 731-8.

6. Singh N, Hofmann TJ, Gershenson Z, Levine BL, Grupp SA, Teachey DT, et al. Monocyte lineage-derived IL-6 does not affect chimeric antigen receptor T-cell function. Cytotherapy. 2017; 19: 867-80.

7. Le RQ, Li L, Yuan W, Shord SS, Nie L, Habtemariam BA, et al. FDA Approval Summary: Tocilizumab for Treatment of Chimeric Antigen Receptor T Cell-Induced Severe or LifeThreatening Cytokine Release Syndrome. Oncologist. 2018; 23: 943-47.

8. Gust J, Hay KA, Hanafi LA, Li D, Myerson D, GonzalezCuyar LF, et al. Endothelial Activation and Blood-Brain Barrier Disruption in Neurotoxicity after Adoptive Immunotherapy with CD19 CAR-T Cells. Cancer Discov. 2017; 7: 140419.

9. Santomasso BD, Park JH, Salloum D, Riviere I, Flynn J, 
Mead E, et al. Clinical and Biological Correlates of Neurotoxicity Associated with CAR T-cell Therapy in Patients with B-cell Acute Lymphoblastic Leukemia. Cancer Discov. 2018; 8: $958-71$.

10. Taraseviciute A, Tkachev V, Ponce R, Turtle CJ, Snyder JM, Liggitt HD, et al. Chimeric Antigen Receptor T CellMediated Neurotoxicity in Nonhuman Primates. Cancer Discov. 2018; 8: 750-63.

11. Jain T, Knezevic A, Pennisi M, Chen Y, Ruiz JD, Purdon TJ, et al. Hematopoietic recovery in patients receiving chimeric antigen receptor T-cell therapy for hematologic malignancies. Blood Adv. 2020; 4: 3776-87.

12. Neelapu SS, Locke FL, Bartlett NL, Lekakis LJ, Miklos DB, Jacobson CA, et al. Axicabtagene Ciloleucel CAR T-Cell Therapy in Refractory Large B-Cell Lymphoma. N Engl J Med. 2017; 377: 2531-44.

13. Elsallab M, Levine BL, Wayne AS, Abou-El-Enein M. CAR T-cell product performance in haematological malignancies before and after marketing authorisation. Lancet Oncol. 2020; 21: e104-16.

14. Reagan PM, Friedberg JW. Axicabtagene ciloleucel and brexucabtagene autoleucel in relapsed and refractory diffuse large B-cell and mantle cell lymphomas. Future Oncol. 2021; 17: $1269-83$.

15. Abramson JS, Palomba ML, Gordon LI, Lunning MA, Wang $\mathrm{M}$, Arnason J, et al. Lisocabtagene maraleucel for patients with relapsed or refractory large B-cell lymphomas (TRANSCEND NHL 001): a multicentre seamless design study. Lancet. 2020; 396: 839-52.

16. Munshi NC, Anderson LD, Jr., Shah N, Madduri D, Berdeja $\mathrm{J}$, Lonial S, et al. Idecabtagene Vicleucel in Relapsed and Refractory Multiple Myeloma. N Engl J Med. 2021; 384: 70516.

17. Lee DW, Gardner R, Porter DL, Louis CU, Ahmed N, Jensen $\mathrm{M}$, et al. Current concepts in the diagnosis and management of cytokine release syndrome. Blood. 2014; 124: 188-95.

18. Porter D, Frey N, Wood PA, Weng Y, Grupp SA. Grading of cytokine release syndrome associated with the CAR $\mathrm{T}$ cell therapy tisagenlecleucel. J Hematol Oncol. 2018; 11: 35.

19. Park JH, Riviere I, Gonen M, Wang X, Senechal B, Curran KJ, et al. Long-Term Follow-up of CD19 CAR Therapy in Acute Lymphoblastic Leukemia. N Engl J Med. 2018; 378: 449-59.

20. Neelapu SS, Tummala S, Kebriaei P, Wierda W, Gutierrez C, Locke FL, et al. Chimeric antigen receptor T-cell therapy assessment and management of toxicities. Nat Rev Clin Oncol. 2018; 15: 47-62.

21. Traube C, Silver G, Kearney J, Patel A, Atkinson TM, Yoon MJ, et al. Cornell Assessment of Pediatric Delirium: a valid, rapid, observational tool for screening delirium in the PICU*. Crit Care Med. 2014; 42: 656-63.

22. Schuster SJ, Bishop MR, Tam CS, Waller EK, Borchmann P, McGuirk JP, et al. Tisagenlecleucel in Adult Relapsed or Re- fractory Diffuse Large B-Cell Lymphoma. N Engl J Med. 2019; 380: 45-56.

23. Greenbaum U, Kebriaei P, Srour SA, Olson A, Bashir Q, Neelapu SS, et al. Chimeric antigen receptor T-cell therapy toxicities. Br J Clin Pharmacol. 2021; 87: 2414-24.

24. Mahadeo KM, Khazal SJ, Abdel-Azim H, Fitzgerald JC, Taraseviciute A, Bollard CM, et al. Management guidelines for paediatric patients receiving chimeric antigen receptor $\mathrm{T}$ cell therapy. Nat Rev Clin Oncol. 2019; 16: 45-63.

25. Henter JI, Horne A, Aricó M, Egeler RM, Filipovich AH, Imashuku S, et al. HLH-2004: Diagnostic and therapeutic guidelines for hemophagocytic lymphohistiocytosis. Pediatr Blood Cancer. 2007; 48: 124-31.

26. Brudno JN, Kochenderfer JN. Toxicities of chimeric antigen receptor T cells: recognition and management. Blood. 2016; 127: $3321-30$.

27. Hill JA, Seo SK. How I prevent infections in patients receiving CD19-targeted chimeric antigen receptor T cells for Bcell malignancies. Blood. 2020; 136: 925-35.

28. Los-Arcos I, Iacoboni G, Aguilar-Guisado M, AlsinaManrique L, Diaz de Heredia C, Fortuny-Guasch C, et al. Recommendations for screening, monitoring, prevention, and prophylaxis of infections in adult and pediatric patients receiving CAR T-cell therapy: a position paper. Infection. 2021; 49: 215-31.

29. Yakoub-Agha I, Chabannon C, Bader P, Basak GW, Bonig H, Ciceri F, et al. Management of adults and children undergoing chimeric antigen receptor T-cell therapy: best practice recommendations of the European Society for Blood and Marrow Transplantation (EBMT) and the Joint Accreditation Committee of ISCT and EBMT (JACIE). Haematologica. 2020; 105: 297-316.

30. Kansagra AJ, Frey NV, Bar M, Laetsch TW, Carpenter PA, Savani BN, et al. Clinical Utilization of Chimeric Antigen Receptor T Cells in B Cell Acute Lymphoblastic Leukemia: An Expert Opinion from the European Society for Blood and Marrow Transplantation and the American Society for Blood and Marrow Transplantation. Biol Blood Marrow Transplant. 2019; 25: e76-85.

31. Schubert ML, Schmitt M, Wang L, Ramos CA, Jordan K, Muller-Tidow C, et al. Side-effect management of chimeric antigen receptor (CAR) T-cell therapy. Ann Oncol. 2021; 32: 34-48.

32. Hill JA, Giralt S, Torgerson TR, Lazarus HM. CAR-T - and a side order of IgG, to go? - Immunoglobulin replacement in patients receiving CAR-T cell therapy. Blood Rev. 2019; 38: 100596.

https://doi.org/10.31547/bct-2021-011

Copyright (C)2021 Asia-Pacific Blood and Marrow Transplantation Group (APBMT). This is an open access article distributed under CC BY-NC license (https://creativecommon s.org/licenses/by-nc/4.0/). 